PROCEEDINGS OF THE

AMERICAN MATHEMATICAL SOCIETY

Volume 128, Number 11, Pages 3445-3451

S 0002-9939(00)05417-4

Article electronically published on May 18, 2000

\title{
DEHN SURGERIES ON STRONGLY INVERTIBLE KNOTS WHICH YIELD LENS SPACES
}

\author{
MIKAMI HIRASAWA AND KOYA SHIMOKAWA
}

(Communicated by Ronald Fintushel)

\begin{abstract}
In this article we show no Dehn surgery on nontrivial strongly invertible knots can yield the lens space $L(2 p, 1)$ for any integer $p$. In order to do that, we determine band attaches to $(2,2 p)$-torus links producing the trivial knot.
\end{abstract}

\section{INTRODUCTION}

Some results are known concerning Dehn surgeries on strongly invertible knots yielding lens spaces. The property $\mathrm{P}$ for strongly invertible knots was confirmed by S. Bleiler and M. Scharlemann [4]. Bleiler and R. Litherland [3] proved that $L(2,1)$, i.e., the real projective 3 -space, cannot be obtained by Dehn surgeries on nontrivial strongly invertible knots. They also conjectured that no nontrivial Dehn surgery on nontrivial knots can yield a lens space $M$ with $\left|\pi_{1}(M)\right|<5$. To prove this conjecture for strongly invertible knots, one has only to consider the case where Dehn surgeries on nontrivial strongly invertible knots yield the lens spaces $L(3,1)$ and $L(4,1)$. In this paper we show that no Dehn surgery on nontrivial strongly invertible knots can yield the lens space $L(2 p, 1)$ for any integer $p$.

S. Wang and Q. Zhou 19 conjectured that no nontrivial Dehn surgery on nontrivial knots can yield a 3 -manifold $M$ with $\left|\pi_{1}(M)\right|<5$. For symmetric knots there are some results on this conjecture. The property $\mathrm{P}$ for symmetric knots was confirmed by M. Culler, C. McA. Gordon, J. Luecke and P. B. Shalen [5]. In [19] it is shown that if a non-torus symmetric knot $K$ admits a nontrivial cyclic surgery yielding $M$, then $K$ is a strongly invertible knot (with no other symmetry) and $M$ is a lens space. We remark that they assume Thurston's orbifold geometrization "theorem" [17], which has not been proved precisely yet. Our result extends to a partial solution to the above conjecture in [19] for symmetric knots modulo Thurston's orbifold geometrization "theorem".

The above conjecture of Bleiler and Litherland is subsumed by the following conjecture of Gordon [9, Problem 1.78]:

"If a Dehn surgery on a knot $K$ yields a lens space, then $K$ is a Berge knot."

Received by the editors November 5, 1997 and, in revised form, January 8, 1999.

2000 Mathematics Subject Classification. Primary 57N10, 57M25.

Key words and phrases. Dehn surgery, strongly invertible knot, lens space, banding.

This research was partially supported by Fellowships of the Japan Society for the Promotion of Science for Japanese Junior Scientists. 
For the definition of Berge knots, see [1] or 9]. If the conjecture holds, then the orders of the fundamental groups of lens spaces obtained by Dehn surgeries on nontrivial knots are at least five. $\left(\frac{-5}{1}\right.$-surgery on the trefoil knot yields $L(5,4)$.) Moreover if the knot is hyperbolic, then it is at least eighteen. $\left(\frac{18}{1}\right.$-surgery on the $(-2,3,7)$-pretzel knot yields $L(18,5)$.)

A knot $K$ in $S^{3}$ is said to be strongly invertible if there is an orientation preserving involution of $S^{3}$ which preserves $K$ as a set and reverses the orientation of $K$. By a result of [18] such an involution is equivalent to a $\pi$-rotation whose axis is unknotted and meets $K$ in exactly two points.

Theorem 1.1. No Dehn surgery on nontrivial strongly invertible knots can yield the lens space $L(2 p, 1)$ for any integer $p$.

Theorem 1.1 has the following two corollaries.

Corollary 1.2. No Dehn surgery on nontrivial strongly invertible knots can yield $L(2 p, q)$ with $\left|\pi_{1}(L(2 p, q))\right| \leq 6$.

If we assume Thurston's orbifold geometrization "theorem", we can generalize Corollary 1.2 by using results of [19].

Corollary 1.3. Thurston's orbifold geometrization "theorem" being assumed, no nontrivial Dehn surgery on nontrivial symmetric knots can yield a 3-manifold $M$ such that $\left|\pi_{1}(M)\right|$ is even and not greater than 6 .

Proof. Suppose that a non-trivial Dehn surgery on a symmetric knot $K$ yields a 3 -manifold $M$ as in Corollary 1.3 Then by [11], $\pi_{1}(M) \cong \mathbb{Z} / 2 p \mathbb{Z}$ where $p=1,2$ or 3. By [19 Theorem and Corollary 1], we see that $K$ is a strongly invertible knot and that $M$ is a lens space, for torus knots are strongly invertible. Hence by Corollary 1.2, we obtain the conclusion.

We prove Theorem 1.1 by analyzing a band attach to a $(2,2 p)$-torus link producing the trivial knot.

Let $L$ be a link in $S^{3}$. Suppose there is a band $b: I \times I \rightarrow S^{3}$ such that $b^{-1}(L)=I \times \partial I$, where $I=[0,1]$ is an interval. Let $L_{b}$ denote the knot or link obtained by replacing $b(I \times \partial I)$ in $L$ with $b(\partial I \times I)$. We call $L_{b}$ a banding of $L$. For simplicity we denote $b(I \times I)$ by $b$.

Note that there is a way to attach a band to a $(2,2 p)$-torus link $L$ producing the trivial knot as in Figure 1.1. We say that a band $b$ is standard if it is isotopic to the one in Figure 1.1. In other words, it is isotopic relative $b \cap L$ to a band in the cabling annulus of $L$ which connects two components of $L$.

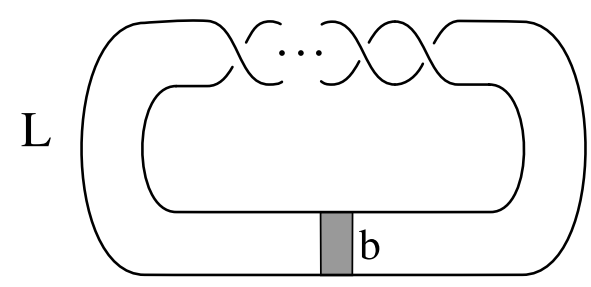

FIGURE 1.1.

We show that the above is the only way to yield the trivial knot: 
Theorem 1.4. Let $L$ be a non-trivial $(2,2 p)$-torus link in $S^{3}$, where $p$ is any integer. If $L_{b}$ is trivial, then $b$ is standard.

A. Thompson studied the case of the Hopf link $((2,2)$-torus link $)$ in [16].

Let $B=S^{3}$-int $N(b)$, where $N(\cdot)$ denotes the regular neighborhood. Then the pair $(B, B \cap L)$ is a 2-string tangle. Theorem 1.4 has a following direct corollary, which we use to prove Theorem 1.1

Corollary 1.5. Let $L$ be a $(2,2 p)$-torus link in $S^{3}$, where $p$ is any integer. If $L_{b}$ is trivial, then $(B, B \cap L)$ is a trivial 2-string tangle.

To prove Theorem 1.4 we consider more general cases. Let $\chi(L)=\max \{\chi(S)\}$, where $\chi(\cdot)$ is the Euler characteristic and $S$ is an oriented surface without closed components such that $\partial S=L$.

Theorem 1.6. Suppose that $L$ and $L_{b}$ have coherent orientations except for the band $b$. Then $\chi(L) \leq \chi\left(L_{b}\right)-1$ if and only if $L$ has a minimal genus Seifert surface $S$ such that $S \supset b$.

Remark 1.7. We note that Theorem 1.6 can also be deduced from the combination of [15, Theorem 1.4] and [15, Proposition 3.1], where they apply Gabai's result in [6]. However in order to clarify the structure of the proof, we give a direct proof also using Gabai's result.

\section{DeHN SURGERIES ON STRONGLY INVERTIBLE KNOTS}

In this section we prove Theorem 1.1 assuming Corollary 1.5 which directly follows from Theorem 1.4 For that purpose, we study integral Dehn surgeries on strongly invertible knots by examining band attaches to links producing the trivial knot. The idea is explained in the proof of Lemma 2.2, where we need the following proposition shown by J. M. Montesinos [12] and Bleiler [2, Theorem 1].

Proposition 2.1. For a strongly invertible knot $K$, the manifold $K\left(\frac{m}{n}\right)$ obtained by an $\frac{m}{n}$-surgery on $K$ double-branch-covers $S^{3}$. The pair of the base space and the branch set $B\left(K ; \frac{m}{n}\right)$ of this covering decomposes into an $\frac{m}{n}$-rational tangle $\left(B_{1}, T_{1}\right)$ and a locally trivial 2-string tangle $\left(B_{2}, T_{2}\right)$ attached together in a manner specified by $\frac{m}{n}$. Moreover if $K$ is nontrivial, then $\left(B_{2}, T_{2}\right)$ is prime.

Here, a 2-string tangle, or a tangle in brief, is a pair $(B, T)$, where $B$ is a 3 -ball and $T$ is a union of two properly embedded arcs. The trivial tangle is a tangle homeomorphic as a pair to $\left(D \times I,\left\{x_{1}, x_{2}\right\} \times I\right)$, where $x_{i}$ 's are points lying in the interior of a disc $D$. A tangle $T$ is said to be non-split if for each properly embedded disc $F$ in $B$ disjoint from $T$, one of the two 3-balls cl $(B-F)$ does not meet $T$. A tangle $T$ is said to be locally trivial if each 2 -sphere in $B$ intersecting $T$ in exactly two points bounds a 3-ball $B^{\prime}$ such that $\left(B^{\prime}, B^{\prime} \cap T\right)$ is the trivial 1-string tangle. A tangle is said to be prime if it is non-split and locally trivial.

Lemma 2.2. No integral Dehn surgery on nontrivial strongly invertible knots can yield the lens space $L(2 p, 1)$ for any integer $p$.

Proof of Lemma 2.2 Suppose $K\left(\frac{m}{n}\right)$ is $L(2 p, 1)$, where $K$ is a nontrivial strongly invertible knot and $|n|=1$. Since the case where $p=0$ has been done in [7, we can assume $p \neq 0$. By [8], $L(p, q)$ double-branch-covers $S^{3}$ in a unique way, where the branch set is a 2-bridge knot or link $S(p, q)$. Hence $B\left(K ; \frac{m}{n}\right)$ is a 2-bridge 
link $S(2 p, 1)$, i.e., a $(2,2 \mathrm{p})$-torus link in $S^{3}$. On the other hand, since $K\left(\frac{1}{0}\right)=$ $S^{3}, B\left(K ; \frac{1}{0}\right)$ is a trivial knot. By Proposition [2.1, $B\left(K ; \frac{1}{0}\right)$ is decomposed into a $\frac{1}{0}$-rational tangle $\left(B_{1}, T_{1}\right)$ and a locally trivial tangle $\left(B_{2}, T_{2}\right)$, and $B\left(K ; \frac{m}{n}\right)$ is decomposed into an $\frac{m}{n}$-rational tangle $\left(B_{1}^{\prime}, T_{1}^{\prime}\right)$ and $\left(B_{2}, T_{2}\right)$. Since $|n|=1, B\left(K ; \frac{1}{0}\right)$ is a banding of $B\left(K ; \frac{m}{n}\right)$. By applying Corollary 1.5, we see that $\left(B_{2}, T_{2}\right)$ is a trivial tangle. Then from Proposition 2.1, it follows that $K$ is trivial, which is a contradiction.

Proof of Theorem 1.1. Suppose $K\left(\frac{m}{n}\right)$ is $L(2 p, 1)$, where $K$ is a nontrivial strongly invertible knot. As in the proof of Lemma 2.2] we assume $p \neq 0$.

Case $1 . K$ is a non-torus knot. In this case, we have $|n|=1$ by [5, Corollary 1]. Hence the conclusion follows from Lemma 2.2 .

Case $2 . K$ is a torus knot. In this case, Lemma 2.3 below justifies the claim.

Lemma 2.3. An $\frac{m}{n}$-surgery on a nontrivial $(r, s)$-torus knot yields a lens space $L(p, 1)$, where $n>0$ and $r>s$, if and only if $(r, s, m, n, p)=(3, \pm 2, \mp 5,1, \mp 5)$.

Proof of Lemma 2.3. Suppose a nontrivial $\frac{m}{n}$-surgery on an $(r, s)$-torus knot yields a lens space. Since $n \neq 0$, we may assume $n>0$. Then under the assumption that $r>s>0$, it is shown in [13] that the obtained lens space is $L\left(|m|, n s^{2}\right)$ and $|r s n+m|=1$.

(Proof of the "if" part.) We can see that $L(5,4) \cong L(-5,1)$ is obtained via $\frac{-5}{1}$-surgery on the $(3,2)$-torus knot. Taking the mirror images, we also see that $L(5,1)$ is obtained via $\frac{5}{1}$-surgery on the $(3,-2)$-torus knot.

(Proof of the "only if" part.) Case 1. $r>s>1$. Suppose a nontrivial surgery on a nontrivial torus knot yielded a lens space $L(p, 1)$. Since $L(-p, 1) \cong L(p,-1)$, we see that $L\left(p^{\prime}, 1\right)$ or $L\left(p^{\prime},-1\right)$ is obtained where $p^{\prime}>0$. Then by Moser's formulae above we have

$$
n s^{2} \equiv \pm 1(\bmod m), \quad r s n+m= \pm 1
$$

Now we eliminate possibilities. From (1), we have $r+\varepsilon s=k m$, for some $k \in \mathbb{Z}-\{0\}$ and $\varepsilon= \pm 1$. Substituting this into the latter part of (1), we have

$$
r s n k+r+\varepsilon s= \pm k, \text { i.e., } k=-\frac{r+\varepsilon s}{r s n \mp 1}<0 .
$$

Now $|r+\varepsilon s|-|r s n \mp 1|=r+\varepsilon s-r s n \pm 1=r(1-s n)+\varepsilon s \pm 1 \leq-r+s+1 \leq 0$. Actually the equalities in the above hold, because $k \in \mathbb{Z}-\{0\}$. Therefore $k=$ $\pm 1, s n=2, \varepsilon=1$ and $r=s+1$. Since $k<0, s>1$ and $n>0$, we have $k=-1, s=2, n=1, r=3$ and hence $m=(r+\varepsilon s) / k=-5$ and $p^{\prime}=|m|=5$. Now that $n s^{2}=4 \equiv-1(\bmod 5)$, we have $L(p, 1) \cong L(5,-1) \cong L(-5,1)$. Therefore $(r, s, m, n, p)=(3,2,-5,1,-5)$.

Case 2. $r>-s>1$. Taking mirror images, we can use the formulae and obtain $(r, s, m, n, p)=(3,-2,5,1,5)$.

Remark 2.4. In [19] it is shown that there is a 'non-standard' way of banding the $(5,2)$-torus knot yielding the trivial knot. The fact that $L( \pm 5,1)$ is obtained from the trefoil is also observed from this. (Apply the Montesinos trick to Figure 4 of [19].) 


\section{BANDing ANd Minimal genus Seifert SURfaces}

In this section we prove Theorems 1.4 and 1.6. First we prove Theorem 1.6.

Proof of Theorem 1.6. (Proof of $(\Leftarrow)$ ) Let $S^{\prime}$ be a Seifert surface for $L_{b}$ obtained by cutting $S$ along the core of the band. Then $\chi\left(S^{\prime}\right)=\chi(S)+1 \leq \chi\left(L_{b}\right)$. Since $S$ is of minimal genus, $\chi(S)=\chi(L)$. Therefore $\chi(L)+1 \leq \chi\left(L_{b}\right)$.

(Proof of $(\Rightarrow)) L$ together with the band $b$ locally appears as in Figure 3.1. Let $k$ be an unknotted circle going once around $b$ spanning a disc that transversely meets $L$ in two points of opposite orientation and meets $b$ in the core of $b$. Let $S$ be a Seifert surface for $L$ which has the minimal genus among those missing $k$. Then we may assume $S \supset b$. Let $M=E\left(S^{3}, L \cup k\right)$. Then the manifold obtained from $M$ by filling in a solid torus along $\partial N(k)$ with slope $\frac{1}{0}$ (resp. 0 ) is $E\left(S^{3}, L\right)$, (resp. $\left.E\left(S^{2} \times S^{1}, L\right)\right)$. Note that we may assume that $S^{3}-(L \cup D)$ is irreducible, by omitting, without loss of generality, split components of $L$. Then by 6 , Corollary 2.4] either (1) or (2) below holds. As argued in [15, proof of Claim 2, p. 530], (2) is equivalent to (3) below, where $S^{\prime}$ is a Seifert surface for $L_{b}$ obtained by cutting $S$ along the core of $b$ as in Figure 3.1.

(1) $S$ is a minimal genus Seifert surface for $L$ in $k\left(\frac{1}{0}\right)=S^{3}$.

(2) $S$ is a minimal genus Seifert surface for $L$ in $k\left(\frac{0}{1}\right)=S^{2} \times S^{1}$.

(3) $S^{\prime}$ is a minimal genus Seifert surface for $L_{b}$ in $S^{3}$.

Case 1. (1) holds. In this case $S \supset b$ and hence the theorem holds.

Case 2. (3) holds. In this case $S^{\prime}$ is of minimal genus and hence $\chi(S)=$ $\chi\left(S^{\prime}\right)-1=\chi\left(L_{b}\right)-1 \geq \chi(L)$. Therefore $\chi(S)=\chi(L)$, i.e., (1) holds.
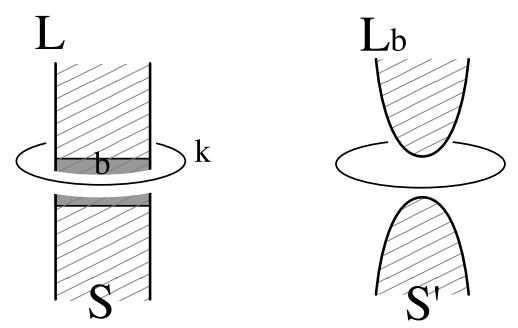

FiguRE 3.1.

To prove Theorem 1.4 by Theorem [1.6] we need the following result of K. Murasugi [14, Lemma 7.1].

Lemma 3.1. $\left|\sigma(L)-\sigma\left(L_{b}\right)\right| \leq 1$.

Proof of Theorem 1.4 Let $L$ be a $(2,2 p)$-torus link, $S$ a minimal genus Seifert surface for $L$ and $b$ a band. Suppose $L_{b}$ is trivial. Then there are two cases according to orientations of $L$. In each case minimal genus Seifert surfaces for $L$ are unique up to isotopy (see for example [10]). Suppose $S$ is the cabling annulus. Then from Theorem 1.6, it follows that $b$ is standard. Now we assume that $S$ is not the cabling annulus, and hence $|p| \geq 2$. We see $|\sigma(L)|=2|p|-1 \geq 3$. On the other hand, $\sigma\left(L_{b}\right)=0$, which contradicts Lemma 3.1.

From Lemma [3.1, we obtain Corollary 3.2 below, which restricts 3-manifolds obtained by integral Dehn surgeries on strongly invertible knots. More specifically, 
Corollary 3.2 restricts the signature of the link $B\left(K ; \frac{m}{n}\right)$ of the surgered manifold $K\left(\frac{m}{n}\right)$, where $|n|=1$ and $B\left(K ; \frac{m}{n}\right)$ is a 2 -component non-split link. We note that Corollary 3.2 applies to broader cases than that of lens spaces. For a 2-component link $L$, let $\sigma^{\prime}(L)$ denote the smallest absolute value of the signatures calculated from all possible orientations of $L$.

Corollary 3.2. Let $K$ be a nontrivial strongly invertible knot and $B\left(K ; \frac{m}{n}\right)$ the branch set of the double branched covering $K\left(\frac{m}{n}\right)$ of $S^{3}$. If $|n|=1$ and $B\left(K ; \frac{m}{n}\right)$ is a 2-component non-split link, then $\sigma^{\prime}\left(B\left(K ; \frac{m}{n}\right)\right) \leq 1$.

Proof. Since $|n|=1$, the trivial knot is a banding of the link $B\left(K ; \frac{m}{n}\right)$. By Lemma 3.1, the corollary follows.

\section{ACKNOWLEDGMENT}

We would like to express our gratitude to the following persons for helpful conversations: Dr. Kazuo Habiro, Professor Koichi Yano, Professor Makoto Sakuma, Professor Tsuyoshi Kobayashi, Professor Chuichiro Hayashi and Dr. Ichiro Torisu.

\section{REFERENCES}

1. J. Berge, Some knots with surgeries yielding lens spaces., preprint.

2. S. Bleiler, Prime tangles and composite knots., In Knot theory and manifolds, Lecture Note in Math. vol. 1144, pp. 1-13 (Springer-Verlag 1983) MR 87e:57006

3. S. Bleiler and R. Litherland, Lens space and Dehn surgery., Proc. Amer. Math. Soc. vol. 107 (1989) 1127-1131. MR 90e:57031

4. S. Bleiler and M. Scharlemann. A projective plane in $R^{4}$ with three critical points is standard. Strongly invertible knots have property P., Topology vol. 27 (1988) 519-540. MR 90e:57006

5. M. Culler, C. McA. Gordon, J. Luecke and P. B. Shalen, Dehn surgery on knots. Ann. of Math. vol. 125 (1987) 237-300. MR 88a:57026

6. D. Gabai, Foliations and the topology of 3-manifolds II., J. Diff. Geom. vol. 26 (1987) 461-478. MR 89a:57014a

7. _ Foliations and the topology of 3-manifolds III., J. Diff. Geom. vol. 26 (1987) 479-536. MR 89a:57014b

8. C. Hodgson and H. Rubinstein, Involutions and isotopies of lens spaces., In Knot theory and manifolds, Lecture Note in Math. vol. 1144 pp. 60-96 (Springer-Verlag 1983) MR 87h:57028

9. R. Kirby ed., Problems in low-dimensional topology., In Geometric Topology, Part 2, Studies in Advanced Mathematics pp. 34-473, (AMS/IP 1997) CMP 98:01

10. T. Kobayashi, Uniqueness of minimal genus Seifert surfaces for links., Topology Appl. vol. 33 (1989) 265-279. [MR 91c:57009

11. J. Milnor, Groups which act on $S^{n}$ without fixed points., Amer. J. Math. vol. 79 (1957) 623-631. MR 19:761d

12. J. M. Montesinos, Surgery on links and double branched covers of $S^{3}$., In Knots, groups and 3-manifolds, Ann. of Math. Studies vol. 84 pp. 227-259 (Princeton University Press 1975) MR 52:1699

13. L. Moser, Elementary surgery along a torus knot., Pacific J. Math. vol. 38 (1971) 737-745. MR 52:4287

14. K. Murasugi, On a certain numerical invariant of link types., Trans. Amer. Math. Soc. vol. 117 (1965) 387-422.

15. M. Scharlemann and A. Thompson, Link genus and Conway moves., Comm. Math. Helv. vol. 64 (1989) 527-535. MR 91b:57006

16. A. Thompson, Knots with unknotting number one are determined by their complements., Topology vol. 28 (1989) 225-230. MR 90f:57011

17. W. Thurston, Three-manifold with symmetry., preprint. 
18. F. Waldhausen, Über Involutionen der 3-Sphäre., Topology vol. 8 (1969) 81-91. |MR 38:5209

19. S. Wang and Q. Zhou, Symmetry of knots and cyclic surgery., Trans. Amer. Math. Soc. vol. 330 (1992) 665-676. MR 92f:57017

Department of Mathematics, Graduate School of Science, Osaka University, ToyONAKA, OSAKA 560-0043, JAPAN

E-mail address: hirasawa@math.sci.osaka-u.ac.jp

Graduate School of Mathematical Sciences, University of Tokyo, Komaba, Tokyo 153-8914, JAPAN

E-mail address: simokawa@poisson.ms.u-tokyo.ac.jp

Current address: Graduate School of Information Sciences, Tohoku University, Katahira Aoba$\mathrm{Ku}$, Sendai 980-8577, Japan

E-mail address: koya@math.is.tohoku.ac.jp 